\title{
A comparative evaluation of different NeuroMyelitis Optica Spectrum Disorder sets criteria
}

\section{Caroline Papeix ( $\nabla$ caroline.papeix@aphp.fr)}

Hopital Universitaire Pitie Salpetriere https://orcid.org/0000-0003-4074-6125

\section{Ysoline Beigneux \\ hôpital de la pitié Salpêtrière}

\section{Elisabeth Maillart}

Hopital Universitaire Pitie Salpetriere

Jérôme de Seze

Hopitaux universitaires de Strasbourg

Catherine Lubetzki

Hopital Universitaire Pitie Salpetriere

\section{Sandra Vukusic}

Hospices Civils de Lyon

Nicolas Collongues

Hopitaux universitaires de Strasbourg

\section{Romain Marignier}

Hospices Civils de Lyon

\section{Short report}

Keywords: Neuromyelitis optica, Multiple sclerosis, criteria

Posted Date: April 2nd, 2020

DOI: https://doi.org/10.21203/rs.3.rs-19442/v1

License: (c) (1) This work is licensed under a Creative Commons Attribution 4.0 International License. Read Full License

Version of Record: A version of this preprint was published at European Journal of Neurology on July 28th, 2020. See the published version at https://doi.org/10.1111/ene.14414. 


\section{Abstract}

Background Three different sets of criteria have been proposed for the diagnosis of neuromyelitis optica spectrum disorders (NMOSD). OBJECTIVE To compare the specificity, sensitivity and diagnostic accuracy of the three different sets of NMOSD criteria, in patients presenting with inflammatory disorders of the central nervous system suggestive of NMOSD. Methods From 236 suspected NMOSD referred for serum AQP4-IgG testing between 2012 and 2014, the three sets of NMOSD criteria (1999, 2006 NMO criteria and 2015 International Panel for NMO Diagnosis criteria) were applied and compared to the final diagnosis. Results Seventy-six patients fulfilled at least one set of criteria and 28 patients fulfilled all NMOSD set of criteria. The final diagnosis was NMOSD in 66 cases, MS according to the MacDonald 2010 in 85 cases and another diagnosis in 85 cases. 2006 NMO criteria has the highest specificity (99\%) and 2015 IPND NMOSD criteria, the highest sensitivity (97\%). For the 1999, 2006 and 2015 IPND NMOSD criteria, the accuracy was respectively $82 \%, 87 \%$ and $97 \%$. Conclusions Our study highlights the limitations of the first set of criteria, that include optico-spinal form of MS. The accuracy of NMO/SD diagnostic criteria improved from 1999 to 2015. It confirms the increased performance of the last set of criteria which covers a larger spectrum of clinical presentation. This study raises some the concern to classify patients with seronegative transverse myelitis or optic neuritis, and MOG-antibody associated disease.

\section{Introduction}

Since 1999, three different sets of diagnostic criteria have been proposed, mainly to distinguish NMO from multiple sclerosis (MS) and other CNS inflammatory disorders. Initially, NMO diagnosis was based essentially on a probabilistic combination of clinical signs, brain or spinal cord MRI and CSF analysis. ${ }^{1}$ The identification of aquaporin-4 immunoglobulin $\mathrm{G}$ antibody (AQP4-IgG) ${ }^{2}$ led to redesign diagnostic criteria in $2006 .{ }^{3}$ In 2015, the International Panel for NMO Diagnosis (IPND) revised the diagnostic criteria for $\mathrm{NMO} / \mathrm{SD}^{4}$, taking into account the broadening clinical spectrum. This new set of criteria allowed an earlier diagnosis by the inclusion of positive AQP4-IgG (AQP4-IgG +) clinically limited forms, and patients with lesions outside the optic nerve and the spinal cord. Analysis of the impact of IPND criteria showed a dramatic increase of the diagnostic yield, in comparison to $2006^{5-7}$, concerning both in AQP4-IgG + and seronegative patients. No direct comparison between the 3 sets of criteria has been performed to date, and studies evaluating the impact of the 2015 IPND criteria, were not designed to assess specificity. Here we compared the diagnostic accuracy and specificity of the 3 sets of NMOSD criteria.

\section{Methods}

\section{Study objective:}

The main objective of this observational retrospective study was to compare the diagnostic value of 3 different sets of NMOSD criteria, as indexed by specificity, sensitivity, and accuracy in patients with clinical symptoms suggestive of NMOSD. 


\section{Study Population}

We evaluated the data of 756 patients (from Lyon, Paris, Strasbourg) referred to the Lyon lab for serum AQP4-IgG testing because of central nervous system inflammatory disorders suspected of being NMOSD (called NMO until 2007) by the treating clinician, between 2012 and 2014. Medical records were available for 520 patients. Incomplete files were excluded from the analysis. Finally, we evaluate 236 files for which all the clinical, imaging and biological information necessary to evaluate the 3 sets of NMOSD criteria were available. For each patients, the presence of the following core clinical characteristics was determined: optic neuritis, myelitis, area postrema syndrome, brainstem syndrome, diencephalic and encephalic syndrome. Brain, spinal cord and orbital MRI were reviewed when available, as well as cerebrospinal fluid (CSF) analyses. All samples were tested for AQP4-IgG using the same technique based on live cell-based assay. When available, MOG-IgG serostatus was collected. ${ }^{8}$

\section{Data collection:}

Clinical, imaging and biological data were collected center by center in 2017 and reviewed by 3 neurologists, expert in NMOSD, from the three participating referral centers. The treating neurologist was defined as the neurologist of the center evaluated and the two evaluating neurologists were defined as external neurologist at the evaluated center.

Blind to the diagnosis proposed by the treating neurologist, the two evaluating neurologists applied retrospectively the 1999, 2006 NMO criteria and 2015 IPND NMOSD criteria ${ }^{1,3,4}$. The final diagnosis was based in 2017, on the conclusion of the treating neurologist.

\section{Statistical analysis:}

Statistical analyses were performed with R software (3.6.0 version). The performance of NMOSD criteria with regard to clinical diagnosis was expressed as sensitivity, specificity, positive predictive value, negative predictive value and accuracy.

\section{Ethical concern}

According to the French legislation regarding observational studies, patients were informed of the study, but did not have to provide a formal and written informed consent to have their anonymized data collected. Data confidentiality and safety were ensured in keeping with the recommendations of the French Commission Nationale Informatique et Libertés, which also provided approval.

\section{Results}

The clinical, imaging, CSF characteristics and AQP4-IgG sero-status of the 236 files analyzed are detailed in Table 1. The final diagnosis was NMOSD in 66 cases, MS in 85 cases. Another diagnosis was made for the 85 other cases (Table 2). 
Among the 236 files analyzed, 76 patients fulfilled at least one set of NMOSD criteria: 37,38 and 70 respectively for the 1999, 2006 and 2015 NMOSD criteria. Forty-seven patients fulfilled only one set of criteria: 7, 10 and 30 respectively for the 1999, 2006 and 2015 NMOSD criteria. Twenty-eight patients fulfilled all NMOSD criteria (Fig. 1).

\section{Comparison between 1999 and 2006 NMOSD criteria}

Twenty-eight patients fulfilled both the 1999 and 2006 NMOSD criteria. Nineteen fulfilled at least one of 1999 or 2006 NMOSD criteria.

Ten patients fulfilled the 2006 but not the 1999 NMOSD criteria. They had clinical signs outside of the optic nerve or spinal cord (6 in area postrema, 5 in brainstem, 1 located in diencephalon). Brain MRI showed "MS like" lesions $(n=3)$ "ADEM like" $(n=1)$, brainstem abnormalities $(n=1)$ and area postrema abnormalities $(n=2)$. Eight patients were tested positive for AQP4-IgG. The diagnosis of NMOSD was made in all of these 10 cases (Table 3).

Nine patients fulfilled 1999 but not the 2006 NMOSD criteria. All patients fulfilling the 1999 criteria but not the 2006 criteria met the major criteria (optic neuritis and acute myelitis and no evidence of clinical disease outside of optic neuritis and spinal cord). As supportive criteria of 1999 NMO/SD criteria, seven had a normal brain MRI, one had a longitudinal extensive transverse myelitis on spinal cord MRI and confluent bilateral subcortical and deep white matter lesions and one had severe and bilateral optic neuritis. None was tested positive for AQP4-IgG. The final diagnosis selected was NMO $(n=4)$, Epstein Barr Virus transverse myelitis and optic neuritis $(n=1)$ and optico-spinal MS $(n=4)$. Among these 4 patients with a final diagnosis of NMO, 2 fulfilled also 2015 IPND criteria. The 2 patients with NMO and fulfilling only 1999 criteria were tested negative for AQP4-lgG. They had bilateral optic neuritis with visual acuity worse than 20/200 (=2 minor supportive 1999 criteria) and short myelitis without other symptoms. Brain MRI fulfilled Mac Donald 2010 criteria for one.

\section{Comparison between 2006 and 2015 NMOSD criteria}

38 patients fulfilled the 2006 and 2015 NMOSD criteria. All patients meeting the 2006 criteria also met the 2015 criteria.

30 patients met the 2015 IPND criteria but not the 2006 or 1999 NMOSD criteria. Twenty-three had a limited form of NMOSD with only optic neuritis $(O N)(n=4)$ or longitudinal extensive transverse myelitis $($ LETM $)(n=19)$. Four cases had also a brainstem $(n=3)$ or area postrema $(n=4)$ syndrome. All limited forms were tested positive for AQP4-IgG. Five of the seven patients with negative AQP4-IgG had at least clinical and radiological area postrema and /or brainstem syndrome. In one case the MRI lesion extended from cervical cord to area postrema (Fig. 2A, patient 52) and in one other case tumefactive brain lesion was observed in addition to brainstem lesion. (Fig. 2B and 2C patient 48).

Among these 30 patients, five (16.6\%) had a final diagnosis other than NMOSD. One had extended brain lesion and LETM with a final diagnosis of endovascular lymphoma (Fig. 3). Two patients were 
considered as MS fulfilling MacDonald 2010 criteria. One patient tested positive for AQP4 with extensive myelitis had a glioma of the spinal cord. Another patient tested positive for AQP4-IgG with optic neuritis was diagnosed as paraneoplastic syndrome. The remaining 25 patients had a final diagnosis of NMOSD, 22 of who were positive for AQP4-IgG.

\section{Final diagnosis}

Among the 66 cases of NMO/SD, 38 were positive for AQP4-IgG and 3 for MOG-IgG (17/28 AQP4-IgG negative patients tested). The clinical, and AQP4-IgG sero-status characteristics of NMOSD patients are detailed in Fig. 4.

Among the 85 cases with a final diagnosis of MS, 7 patients fulfilled at least 1 NMOSD criteria, including onepatient fulfilling the three sets of criteria. One patient with progressive MS was tested positive for AQP4- IgG.

Among the 85 cases without NMO/SD or MS diagnosis (Table 2), 6 patients with isolated myelitis were tested positive for MOG-IgG. Finally, and 34 patients still remained without diagnosis at the date of the evaluation: 16 had isolated or recurrent idiopathic optic neuritis, 15 isolated or recurrent idiopathic transverse myelitis, 1 isolated encephalitis and 2 multiple symptoms.

Value of the three different sets of NMOSD criteria

The specificity of the criteria was highest ( $99 \%$ ) in 2006 NMO criteria. The specificity was similar in the first and the last set of criteria ( $96 \%$ in 1999, and in 2015 IPND criteria). The sensitivity was highest ( 97\%) in 2015 IPND NMOSD criteria and the accuracy significantly increased from the first to the last NMOSD criteria (from 82-97\%) (Table 3).

\section{Discussion}

The accuracy of the NMO sets criteria increases with the successive version of criteria with a significant improvement of the sensitivity from 1999 to 2015 IPND criteria while the specificity remains similar.

The results of this study emphasize the limitation of the 1999 NMO criteria. The 1999 set of criteria allowed the inclusion of optico-spinal form of MS, having therefore a low specificity. The integration of serological status for AQP4 $\operatorname{lgG}^{2}$ in the revised 2006 criteria led to improve drastically the specificity of the NMO set criteria. 2006 criteria, allowing cases with extra optico-spinal presentation to be included, also improved the sensitivity and enlarged the spectrum of the disease.

Our study confirms the increase of diagnostic yields of 2015 IPND criteria in comparison to 2006, due to inclusion of positive AQP4-IgG patients with a limited form of the disease. The 2015 IPND criteria also 
result in inclusion of patients, with either positive or negative AQP4 status, presenting with non opticospinal syndrome (brain, brainstem or diencephalic lesions) in both.

The study also raises some concerns about 2015 IPND criteria with a lower specificity in comparison to 2006. Indeed, some patients fulfilling 2015 IPND criteria and not 2006 NMOSD criteria had an alternative $\mathrm{NMO} / \mathrm{SD}$ diagnosis. This issue concerns mainly patients with brain lesion and negative AQP4-IgG status. Moreover, if 2015 IPND criteria are more accurate than 1999 criteria to distinguish NMOSD from MS there is still matter of concern in the patients with MS like lesions. The appearance of "MS like" brain lesions during the evolution of the disease is particularly worrying in the limited form.

Despite the fact that IPND 2015 allowed an early diagnosis, some patients with NMO related disorder, with either isolated or recurrent TM and ON but tested negative for AQP4-IgG are still not classified. As found in our study, some of these patients are tested positive for MOG-IgG, highlighting the current need to establish a clear definition of MOGAD (MOG - Auto Antibody disease) and its link with NMOSD.

\section{Declarations}

\section{Ethical Approval and Consent to participate}

According to the French legislation regarding observational studies, patients were informed of the study, but did not have to provide a formal and written informed consent to have their anonymized data collected. Data confidentiality and safety were ensured in keeping with the recommendations of the French Commission Nationale Informatique et Libertés, which also provided approval.

Consent for publication : Not applicable

\section{Availability of supporting data}

Because of national confidentiality requirements, only anonymized data, not pseudonymized data, can be shared. While anonymization techniques might result in the impoverishment of data (article 29 of Directive 95/46 /EC, Opinion 04/2014 on Anonymization Techniques -0829/14/EN WP 216) data used for this study were only pseudonymized.

\section{Competing interest}

The authors declare that they have no competing interests. No disclosure relevant to the manuscript.

\section{Funding}

No funding statement to declare for this work.

\section{Author's contributions}


CP, NC, RM designed and conceptualized the study, have a major role in the acquisition of data and analyzed the data and drafted the manuscript for intellectual content. YB Analyzed data; revised the manuscript for intellectual content. All authors listed have revised the manuscript for intellectual contribution to the work. All authors read and approved the final draft for publication.

Acknowledgements : Not applicable

\section{References}

1. Wingerchuk, D. M., Hogancamp, W. F., O'Brien, P. C. \& Weinshenker, B. G. The clinical course of neuromyelitis optica (Devic's syndrome). Neurology 53, 1107-1114 (1999).

2. Lennon, V. A. et al. A serum autoantibody marker of neuromyelitis optica: distinction from multiple sclerosis. Lancet Lond. Engl. 364, 2106-2112 (2004).

3. Wingerchuk, D. M., Lennon, V. A., Pittock, S. J., Lucchinetti, C. F. \& Weinshenker, B. G. Revised diagnostic criteria for neuromyelitis optica. Neurology 66, 1485-1489 (2006).

4. Wingerchuk, D. M. et al. International consensus diagnostic criteria for neuromyelitis optica spectrum disorders. Neurology 85, 177-189 (2015).

5. Hamid, S. H., Elsone, L., Mutch, K., Solomon, T. \& Jacob, A. The impact of 2015 neuromyelitis optica spectrum disorders criteria on diagnostic rates. Mult. Scler. Houndmills Basingstoke Engl. 23, 228233 (2017).

6. Sepúlveda, M. et al. Neuromyelitis optica spectrum disorders: Comparison according to the phenotype and serostatus. Neurol. Neuroimmunol. Neuroinflammation 3, e225 (2016).

7. Hyun, J.-W., Jeong, I. H., Joung, A., Kim, S.-H. \& Kim, H. J. Evaluation of the 2015 diagnostic criteria for neuromyelitis optica spectrum disorder. Neurology 86, 1772-1779 (2016).

8. Marignier, R. et al. Aquaporin-4 antibody-negative neuromyelitis optica: distinct assay sensitivitydependent entity. Neurology 80, 2194-2200 (2013).

9. Polman, C. H. et al. Diagnostic criteria for multiple sclerosis: 2010 Revisions to the McDonald criteria. Ann. Neurol. 69, 292-302 (2011).

\section{Tables}

Table 1: Clinical, imaging, CSF characteristics, AQP4-IgG sero-status of all the patients included 


\begin{tabular}{|c|c|c|c|}
\hline Clinical characteristics & $\begin{array}{l}\text { All patients } \\
\mathrm{N}=236\end{array}$ & $\begin{array}{l}\text { MS patients (final diagnosis) } \\
\mathrm{N}=85\end{array}$ & $\begin{array}{l}\text { NMO patients } \\
\text { (final diagnosis) } \\
\mathrm{N}=66\end{array}$ \\
\hline Transverse myelitis & $149 / 223(66 \%)$ & $52 / 85(61 \%)$ & $63 / 66(95 \%)$ \\
\hline Optic neuritis & $121 / 229(53 \%)$ & $46 / 85(54 \%)$ & $48 / 66(72 \%)$ \\
\hline Area postrema syndrome & $14 / 196(7 \%)$ & $0 / 75(0 \%)$ & $13 / 56(23 \%)$ \\
\hline Brainstem & $35 / 201(17 \%)$ & $14 / 76(18 \%)$ & $13 / 56(23 \%)$ \\
\hline Supratentorial Brain & $32 / 196(16 \%)$ & $19 / 75(25 \%)$ & $4 / 56(7 \%)$ \\
\hline Brain MRI & $230 / 236$ & $83 / 85$ & $65 / 66$ \\
\hline Normal & $125(54 \%)$ & $20(24 \%)$ & $45(69 \%)$ \\
\hline Demyelinating & $95(41 \%)$ & $61(73 \%)$ & $19(29 \%)$ \\
\hline Abnormal but without demyelinating lesions & $10(2 \%)$ & $2(2 \%)$ & $1(2 \%)$ \\
\hline Medullar MRI & $212 / 236$ & 76 & $65 / 66$ \\
\hline Normal & $56(26 \%)$ & $19(25 \%)$ & $4(6 \%)$ \\
\hline Short myelitis (single or multiple) & $88(42 \%)$ & $49(64 \%)$ & $18(28 \%)$ \\
\hline Extensive myelitis & $68(32 \%)$ & $8(11 \%)$ & $43(66 \%)$ \\
\hline Oligoclonal Bands & $91 / 210(43 \%)$ & $66 / 79(84 \%)$ & $12 / 58(21 \%)$ \\
\hline positive AQP4-IgG sero-status & $2 / 85$ & $1 / 85(0.01 \%)$ & $38 / 66(58 \%)$ \\
\hline
\end{tabular}

Table 2: Main clinical characteristic and the final diagnosis of the 85 patients without NMOSD or MS 


\begin{tabular}{|c|c|c|c|}
\hline Clinical characteristics and causes & Number of patients & Clinical characteristics and causes & Number of patients \\
\hline Isolated Optic neuritis & 25 & Isolated Area postrema syndrome & 1 \\
\hline Idiopathic optic neuritis & 10 & Listeriosis & 1 \\
\hline CRION/RION & 4 & Isolated Brain symptoms & 11 \\
\hline Neuro-papillitis & 2 & Clippers & 2 \\
\hline Idiopathic intracranial hypertension & 2 & Unknown & 1 \\
\hline Leber disease & 2 & ADEM & 2 \\
\hline Sarcoidosis & 1 & Behcet's disease & 1 \\
\hline Toxocarosis & 1 & Endovascular lymphoma & 1 \\
\hline Paraneoplasic syndrome & 1 & Paraneoplasic encephalitis & 1 \\
\hline Alcohol optic neuropathy & 1 & Viral meningoencephalitis & 2 \\
\hline Somatoform disorder & 1 & Metabolic leucoencephalopathy & 1 \\
\hline Isolated Myelitis & 33 & Multiple clinical characteristics & 5 \\
\hline Idiopathic & 12 & Endovascular lymphoma & 1 \\
\hline Myelitis with MOG antibody & 6 & Epstein-Barr virus & 1 \\
\hline Ischemic myelitis & 2 & Sjögren's syndrome & 1 \\
\hline Tumor myelitis & 3 & No diagnosis & 2 \\
\hline Lupus & 1 & & \\
\hline Sjögren 's syndrome & 1 & Without suggestive symptom of NMOSD & 10 \\
\hline Sarcoidosis & 2 & Idiopathic intracranial hypertension & 3 \\
\hline Syringomyelia & 1 & Antiphospholipid syndrome & 1 \\
\hline Paraneoplasic myelitis & 1 & Meningitis & 1 \\
\hline Compressive myelopathy & 1 & Myelopathy & 2 \\
\hline Meningoradiculitis & 1 & Lyme disease & 1 \\
\hline Herpetic myelitis & 1 & Somatoform disorder & 1 \\
\hline Listeriosis & 1 & Serous chorioretinitis & 1 \\
\hline
\end{tabular}

Table 3: sensitivity, specificity, positive predictive value, negative predictive value and accuracy of the NMO/SD criteria sets

W1999 Wingerchuk 1999 NMO criteria ${ }^{1}$; W2006: Wingerchuk 2006 NMO criteria ${ }^{3}$; 2015 IPND: 2015 International Panel for NMO Diagnosis ${ }^{4}$ 


\begin{tabular}{|l|l|l|l|l|l|}
\hline & Sensitivity & Specificity & Positive predictive value & Negative predictive value & Accuracy \\
\hline W 1999 & 47 & 96 & 84 & 82 & 82 \\
\hline W 2006 & 56 & 99 & 97 & 85 & 87 \\
\hline IPND 2015 & 97 & 96 & 91 & 99 & 97 \\
\hline
\end{tabular}

\section{Figures}

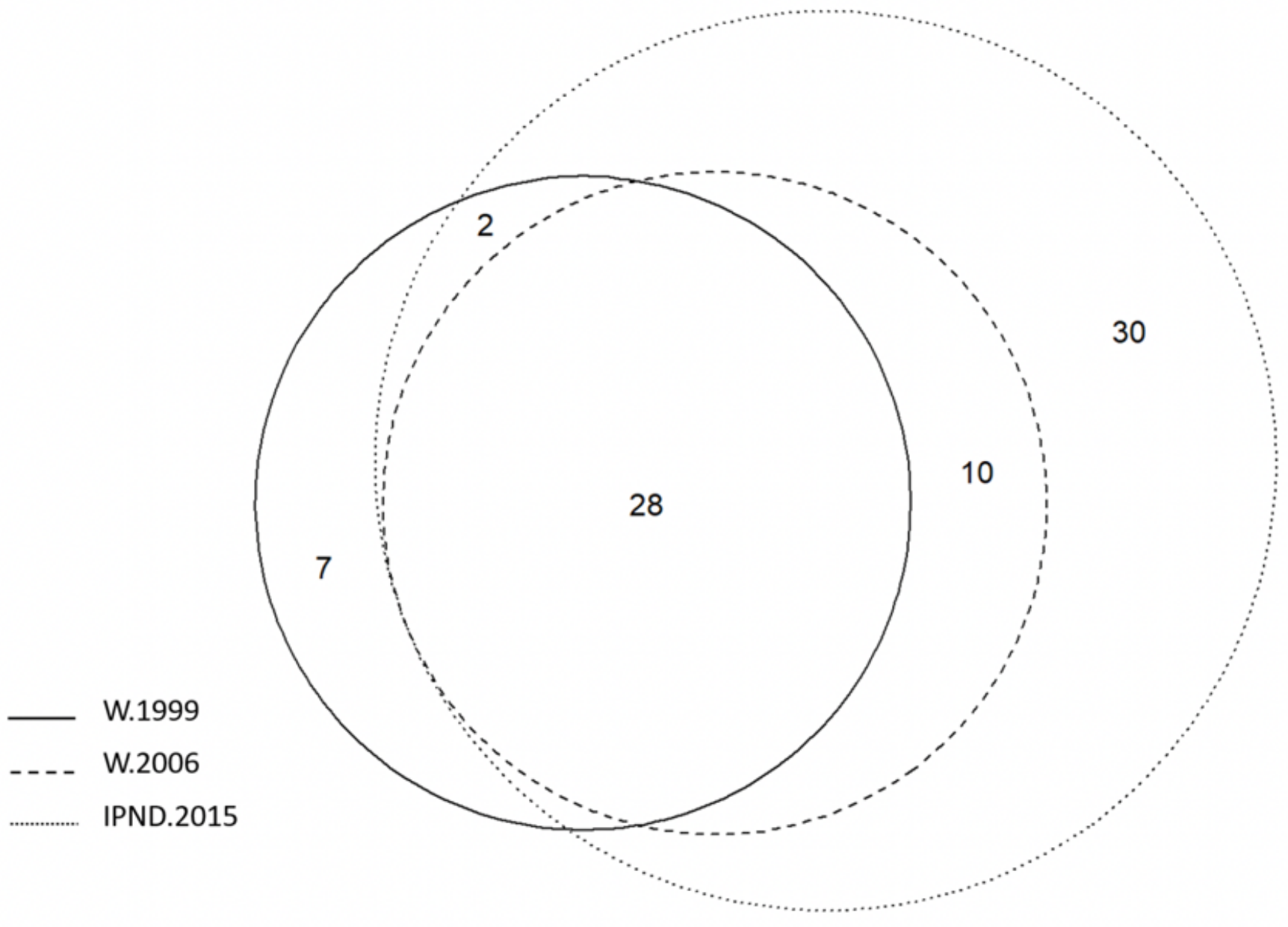

\section{Figure 1}

Venn diagram of different NMO criteria fulfilled by the patients of the study. W.1999=1999 Wingerchuk NMO/SD criteria1; W2006: 2006 Wingerchuk NMO/SD criteria3 ; IPND 2015 : International Panel for NMO Diagnosis 2015430 patients fulfilled only 2015 IPND diagnostic criteria, 10 fulfilled 2006 NMO/SD criteria and 2015 IPND criteria, 28 fulfilled all criteria, 2 fulfilled 2015 IPND criteria and 1999 NMO/SD criteria, 7 fulfilled only 1999 NMO/SD criteria. 


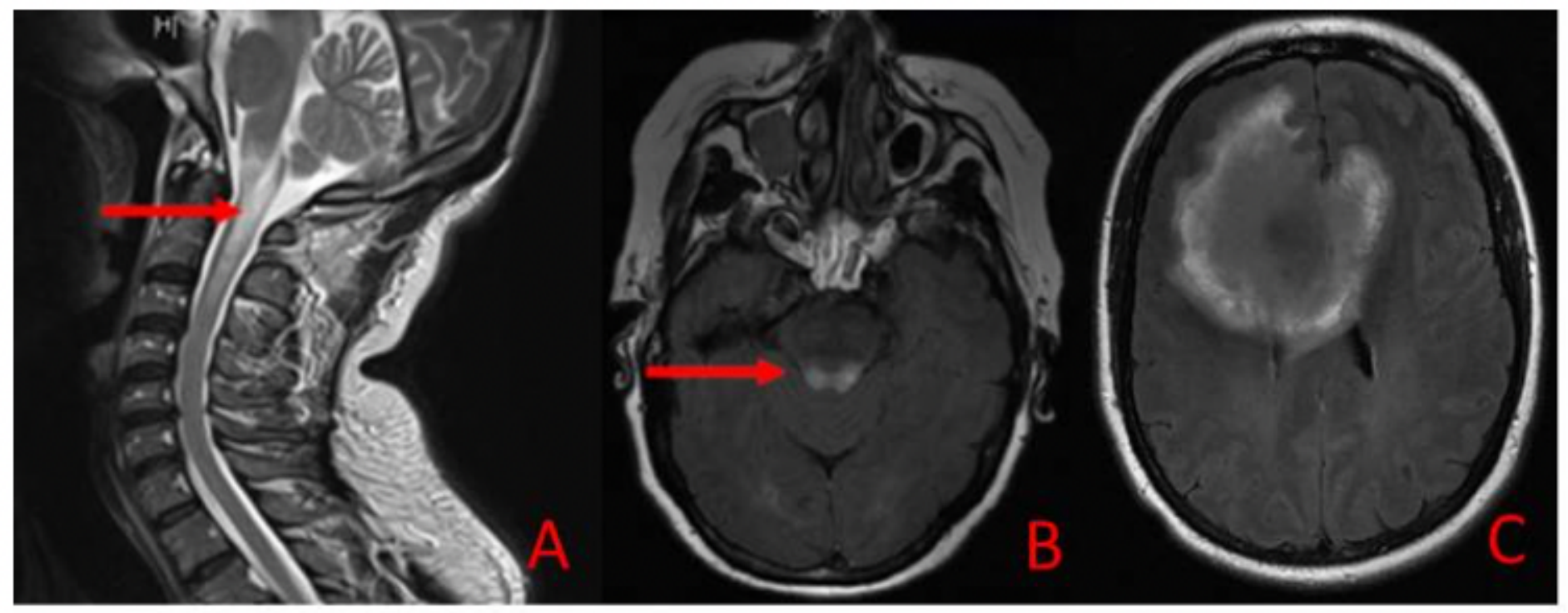

\section{Figure 2}

illustrative cases of patients meeting the 2015 IPND criteria but not the 2006 and 1999 NMO/SD criteria and having negative AQP4-IgG. (Figure 2A, patient 52) T2 Weighted cervical cord MRI shows a lesion extended from cervical cord to area postrema and (Figure 2B and 2C patient 48) FLAIR sequence of cervical cord MRI shows a tumefactive brain lesion in addition to brainstem lesion.

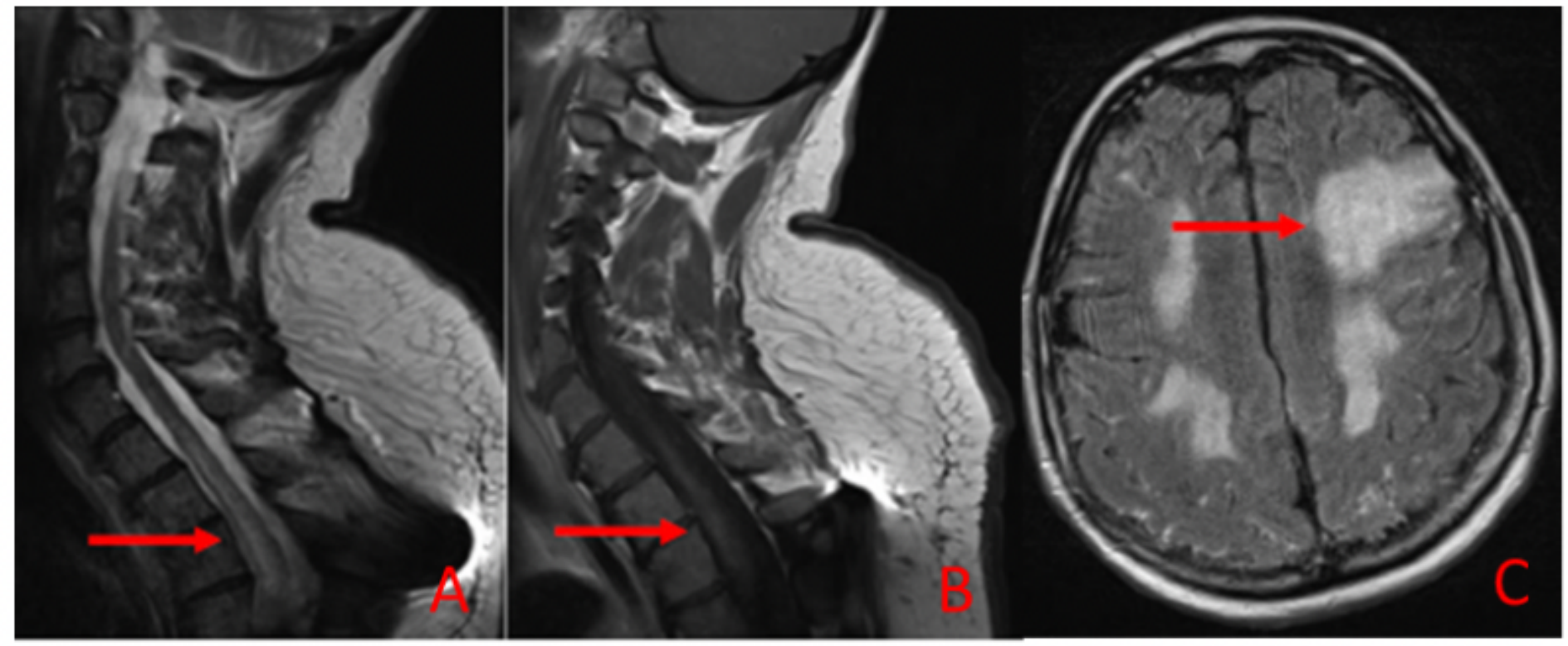

\section{Figure 3}

illustrative case of a patient meeting the 2015 criteria but not the 2006 NMO/SD criteria, and having a final diagnosis of endovascular lymphoma. Figure 3 (A) T2 weighted cervical cord MRI shows a lesion extended from thoracic cord; (B) T1 Weighted cervical cord MRI shows an extended gadolinium enhancement; (C) FLAIR sequence of brain MRI shows a bilateral and extended high-signal. 


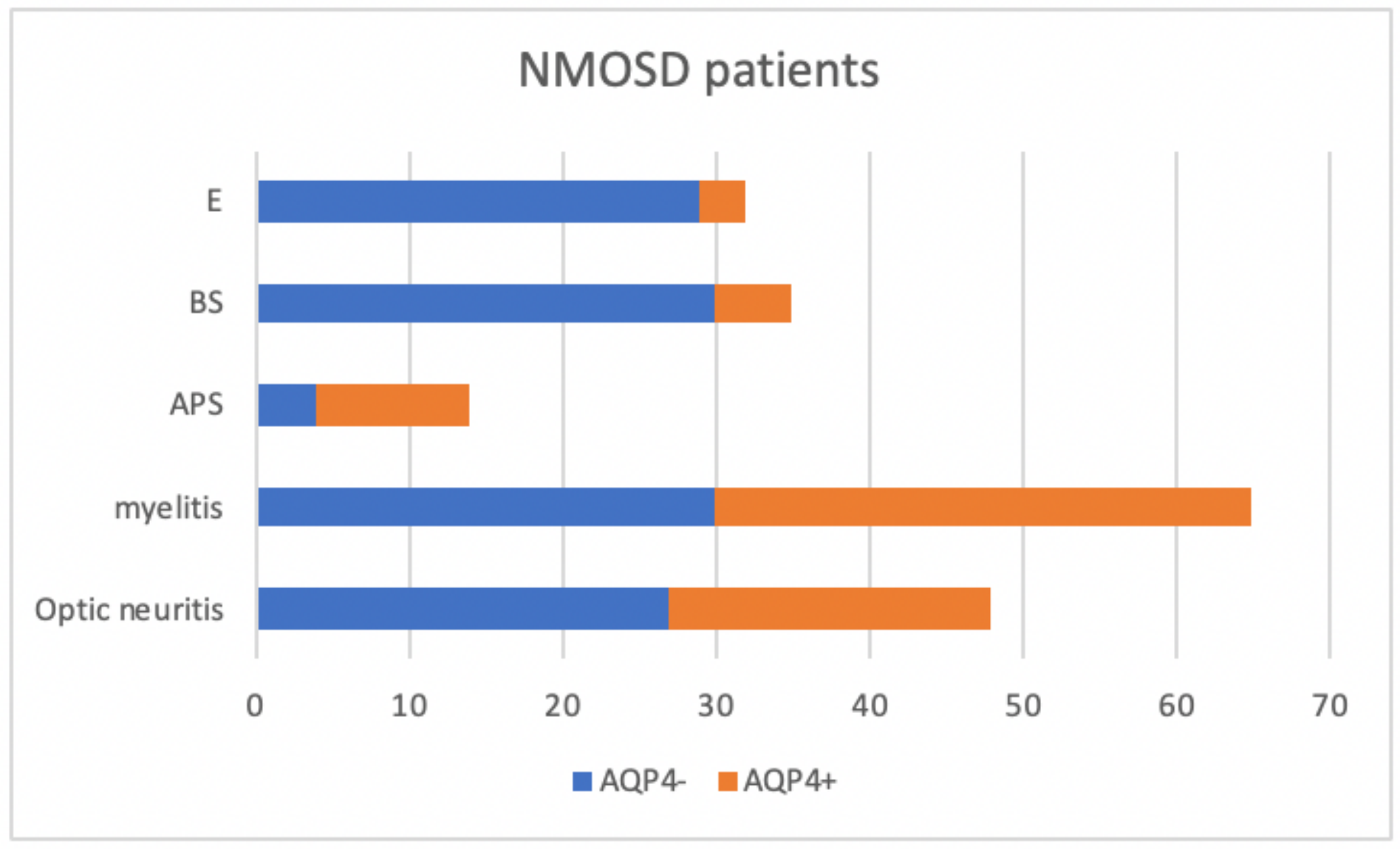

\section{Figure 4}

Clinical characteristics of NMOSD patients according to AQP4 serostatus. E : Encephalitis, BS : BrainStem, APS : Area PoStrema 